\title{
Gadolinium-Doped Iron Oxide Nanoprobe as Multifunctional Bioimaging Agent and Drug Delivery System
}

\author{
Guilong Zhang, Ruohong Du, Lele Zhang, Dongqing Cai, Xiao Sun, Yong Zhou, \\ Jian Zhou, Junchao Qian, Kai Zhong, Kang Zheng, Darnell Kaigler, Wenqing Liu, \\ Xin Zhang,* Duohong Zou, * and Zhengyan Wu*
}

In this study, a high-performance $T_{1}-T_{2}$ dual-model contrast agent by gadolinium-doped iron oxide nanoparticle (GION) is developed. Following its development, the application of this agent in vivo by combining doxorubicin (DOX) and folic acid (FA) (FA-GION-DOX) for targeted drug delivery to monitor cancer treatment is explored. GION showed transverse and longitudinal relaxivities up to $182.7 \times 10^{-3}$ and $7.87 \times 10^{-3} \mathrm{M}^{-1} \mathrm{~s}^{-1}$, respectively, upon $\mathrm{Gd} / \mathrm{Fe}$ ratio in GION at 1/4. DOX released from FA-GION-DOX is $\mathrm{pH}$ dependent and only kills cancer cell after FA receptor-mediated internalization into the acidic environment of endosomes and lysosomes. Systemic delivery of FA-GION-DOX significantly inhibits the growth of tumors and shows good magnetic resonance enhancement in a human cervical cancer xenograft model. Thus, FA-GION-DOX has a potential application for the targeted and magnetic resonance imaging guided therapy of cervical cancer.

\section{Introduction}

Current cancer therapies have attracted wide attention and have had promising success. ${ }^{[1-3]}$ However, these therapies still have limitations such as (1) inability to bypass biological barriers, (2) nontargeted delivery and passive distribution of drugs,

(3) ineffectiveness against metastatic disease, and (4) lack of an effective modality for treatment monitoring. ${ }^{[4-7]}$ Over the past 20 years, the development of nanotechnology has provided some potential methods to overcome these challenges. An example of such a methodology utilizes a single nanomedicine for the dual purpose of targeted drug delivery and in vivo drug monitoring. ${ }^{[8-10]}$

Magnetic resonance imaging (MRI) has become a powerful noninvasive tool in the clinical diagnosis of disease and in vivo imaging through the ability to acquire 3D tomographic images with high spatial resolution. ${ }^{[11,12]}$ Contrast agents are an important consideration when using MRI; however, most clinical contrast agents such as gadolinium chelated diethylenetriaminopentaacetic acid (Gd-DTPA) are small, nontargeted compounds. As such, they sometimes passively distribute into the interstitial spaces of tissues and organs, resulting in an insufficient signal enhancement. With the development of new contrast agents, magnetic nanomaterials have emerged as ideal agents for disease diagnosis and therapy because they can also be used as magnetic-field-guided drug delivery vehicles. ${ }^{[13,14]}$
G. Zhang, R. Du, Dr. D. Cai, X. Sun, Prof. W. Liu, Prof. Z. Wu

Key Laboratory of Ion Beam Bioengineering

Hefei Institutes of Physical Science

Chinese Academy of Sciences and Anhui Province

Hefei 230031, P. R. China

E-mail: zywu@ipp.ac.cn

G. Zhang, R. Du, Dr. D. Cai, X. Sun, Prof. W. Liu, Prof. Z. Wu University of Science and Technology of China

Hefei 230026, P. R. China

R. Du, Y. Zhou, Prof. J. Zhou, Dr. D. Zou

Department of Dental Implant Center

Stomatologic Hospital and College

Key Laboratory of Oral Diseases Research of Anhui Province

Anhui Medical University

Hefei 230032, P. R. China

E-mail: zouduohongyy@126.com

\author{
L. Zhang, Prof. X. Zhang \\ School of Life Sciences \\ Anhui Agricultural University \\ Hefei 230036, P. R. China \\ E-mail: xinzhang@ahau.edu.cn \\ J. Qian, Prof. K. Zhong \\ High Magnetic Field Laboratory \\ Hefei Institutes of Physical Science \\ Chinese Academy of Sciences \\ Hefei, Anhui 230031, P. R. China \\ Dr. K. Zheng \\ Key Laboratory of Materials Physics \\ Institute of Solid State Physics \\ Chinese Academy of Sciences \\ Hefei 230031, P. R. China \\ Dr. D. Kaigler \\ Department of Periodontics and Oral Medicine \\ Department of Biomedical Engineering \\ University of Michigan \\ Ann Arbor, MI 48109, USA
}


Iron oxide nanoparticles (IONs) such as $\mathrm{Fe}_{3} \mathrm{O}_{4}$ serve as good contrast agents ( $T_{2}$-weighted) due to their biocompatibility and magnetic properties. ${ }^{[15-17]}$ However, $T_{2}$-weighted dark signals gained with IONs are easily disturbed by signals from calcification, bleeding, or metal deposits, and these artifacts can impact background imaging. ${ }^{[18-20]}$ As a result, the development of multifunctional imaging agents (such as $T_{1}-T_{2}$ dual-modal contrast agent) for obtaining more accurate diagnostic images is needed.[21] In order to address this issue, recent efforts have been made to introduce appropriate atoms (such as lanthanide ions) into the ION to obtain $T_{1}$-contrast enhancement because lanthanide ions possess more unpaired electrons in their f-electronic orbit. ${ }^{[22-25]}$ However, doped particles which possess these physical and chemical characteristics have not been found to be significantly different from the parent, undoped ION. ${ }^{[26]}$ Thus, alternative doping ratios and atoms need to be further investigated to acquire high efficient $T_{1}-T_{2}$ dual-modal contrast agents for MRI.

Another key objective in the development of IONs is to produce them to serve as targeted drug delivery vehicles, specifically to enhance therapeutic efficiency of cancer drugs. Over the past decade, many nanocarriers, including organic, inorganic, and hybrid ones, have been developed as targeted drug delivery systems for transporting therapeutic agents to disease sites. ${ }^{[7,27,28]}$ The intrinsic instable nature of organic systems precludes on their clinical use. In contrast, IONs have clinical promise because of their capacity to simultaneously serve dual roles: as targeted vehicles for controlled release of drugs and as imaging probes for MRI. ${ }^{[29]}$
In this work, we designed a multifunctional gadoliniumdoped iron oxide nanoparticle (GION) which had highly specific imaging properties and folic acid (FA) receptor specificity for targeted drug delivery. Doxorubicin (DOX, a clinical anticancer drug) was chosen as the model drug and loaded in GION. The results showed that DOX was released from this FA-GIONDOX system only after receptor-mediated internalization into the acidic environment of endosomes and lysosomes. Systemic delivery of FA-GION-DOX significantly inhibited the growth of tumors and showed good MR enhancement in a human cervical cancer xenograft model.

\section{Results and Discussion}

\subsection{Synthesis and Characterization of GION}

As shown in step 1 of Scheme 1, GION was fabricated by thermal decomposition of iron acetylacetonate and gadolinium acetylacetonate complexes in polyethylene glycol and ethylene glycol, which contained polyethylenimine (PEI) as a surfactant. Transmission electron microscopy (TEM) images showed that the as-synthesized GION was nearly monodisperse nanocrystal (Figure $1 \mathrm{a}-\mathrm{c}$ ). In addition, Fe/Gd ratio in the GIONs could be adjusted by controlling the molar ratio of the precursors, and the content of Fe and $\mathrm{Gd}$ elements was determined by inductively coupled plasma mass spectrometry (ICP-MS). Interestingly, we found that the size of GION gradually decreased with increasing amounts of Gd atoms (Figure S1, Supporting Information).

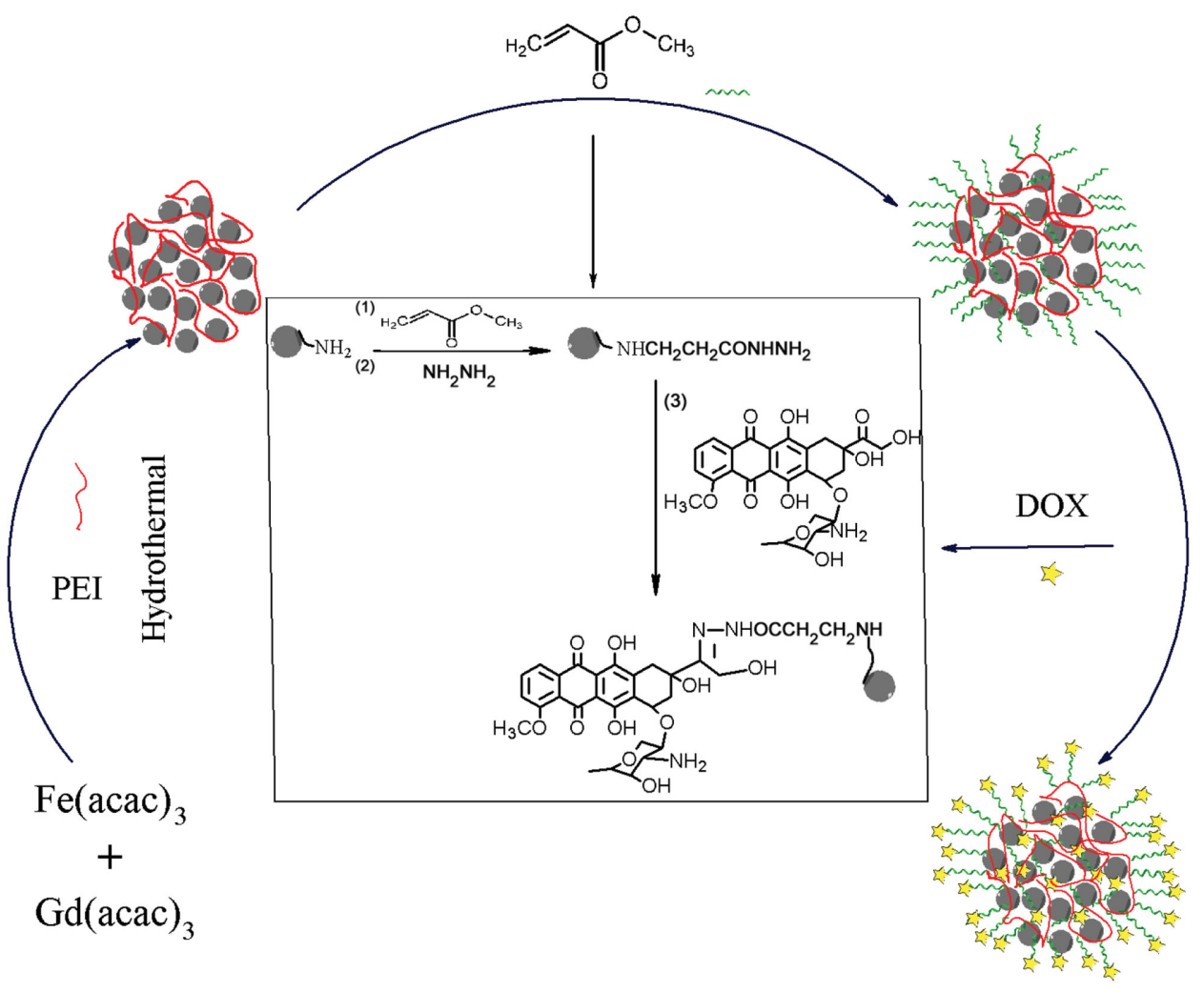

Scheme 1. Schematic illustration of the synthetic procedure for FA-GION-DOX. 

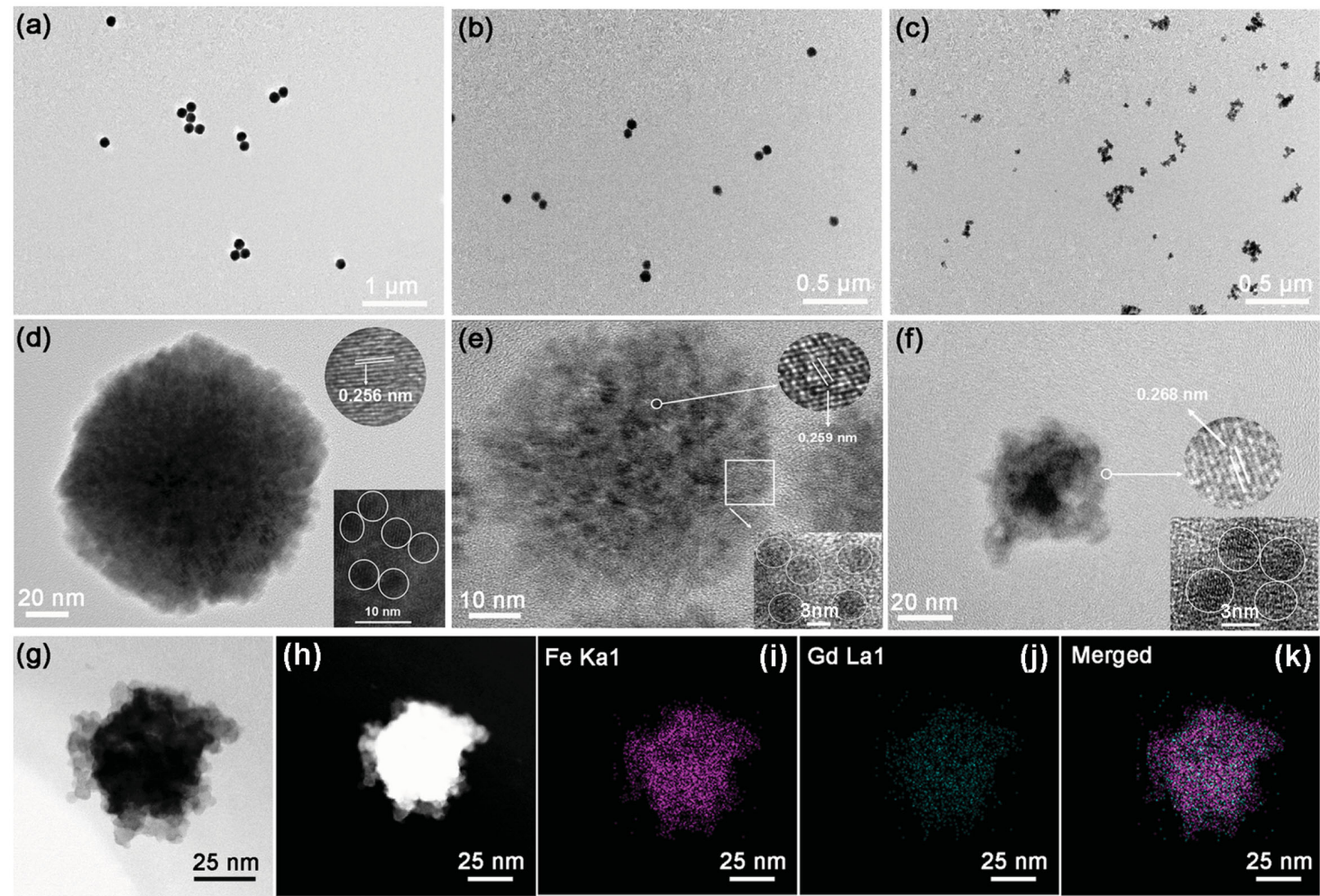

(j) Merged
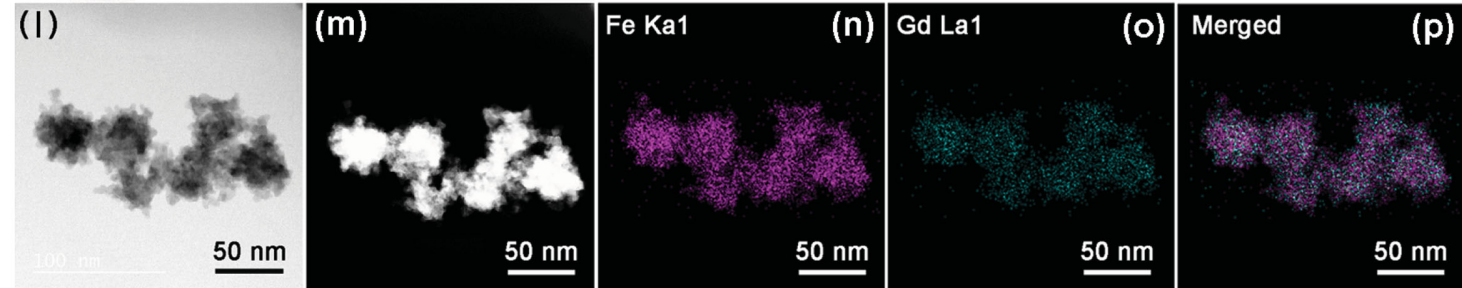

Figure 1. TEM and HRTEM images of a,d) $\left.\mathrm{Fe}_{3} \mathrm{O}_{4}, \mathrm{~b}, \mathrm{e}\right) \mathrm{Gd}_{0.06} \mathrm{Fe}_{2.94} \mathrm{O}_{4}$, and c,f) $\mathrm{Gd}_{0.6} \mathrm{Fe}_{2.4} \mathrm{O}_{4}$. Scanning TEM images in g,l) bright field and h,m) dark field, and the corresponding element area mappings of $\mathrm{i}-\mathrm{k}, \mathrm{n}-\mathrm{p}) \mathrm{Gd}_{0.6} \mathrm{Fe}_{2.4} \mathrm{O}_{4}$.

From the high resolution TEM (HRTEM) images of a single GION nanosphere (insets located in the bottom right of Figure $1 \mathrm{~d}-\mathrm{f}$ ), it could be seen that the nanosphere was composed of lots of tiny nanocrystals with almost same sizes of $5 \mathrm{~nm}$, indicating this nanosphere is a type of superparticle. ${ }^{[30-32]}$ As shown in Figure 1d, the distance between two adjacent planes of $\mathrm{Fe}_{3} \mathrm{O}_{4}$ nanocrystal was measured to be $0.256 \mathrm{~nm}$, corresponding to the (311) plane. However, once Gd ions were doped into crystal lattice, the distance between these adjacent planes began to increase gradually (Figure 1e,f). The energydispersive X-ray element mapping and face scanning analysis indicated that the distribution of Gd and Fe elements in GION was homogeneous (Figure 1g,h and Figures S2-S3, Supporting Information).

X-ray diffraction (XRD) was used to confirm the crystalline structure of GION, and it exhibited obvious diffraction peaks assigned to the spinel structure of magnetite, which were different from the $\mathrm{Gd}_{2} \mathrm{O}_{3}$ simply embedded in ION because in that case obvious diffraction peaks of cubic $\mathrm{Gd}_{2} \mathrm{O}_{3}$ would certainly appear. In addition, compared to $\mathrm{Fe}_{3} \mathrm{O}_{4}$, the diffraction peaks of GION shift to the left, indicating that the interplanar spacing distance of spinelle enlarged because of doping $\mathrm{Gd}$ ions. On the other hand, in Figure 2a, lattice distortion of GION increased with the content of the Gd element in ION, which could be explained that the larger size of the Gd atom occupied the site of the Fe atom. ${ }^{[33,34]}$ X-ray photoelectron spectroscopy (XPS) analysis confirmed the presence of $\mathrm{Fe}$ and $\mathrm{Gd}$, $\mathrm{O}, \mathrm{C}$, and $\mathrm{N}$ in the GION (Figure 2b). Further analysis of the Fe2p peaks in the XPS spectrum of GION showed the Fe2p1/2 and $\mathrm{Fe} 2 \mathrm{p} 3 / 2$ peaks at 711.4 and $724.6 \mathrm{eV}$ (Figure 2c), respectively, thus confirming the presence of $\mathrm{Fe}(\mathrm{III})$. The presence of Gd(III) was also confirmed by the Gd4p peaks at 142.6 and $157.9 \mathrm{eV}$.

\subsection{Magnetic Property and MRI Performance}

Field-dependent magnetization $(M / H)$ curves of $\mathrm{Fe}_{3} \mathrm{O}_{4}$ showed saturation magnetization moments $(\mathrm{Ms})$ were about $79.5 \mathrm{emu} \mathrm{g}^{-1}$ at $300 \mathrm{~K}$ (Figure 3a). However, the saturated magnetization of GION with different $\mathrm{Gd} / \mathrm{Fe}$ ratios was much lower than that of $\mathrm{Fe}_{3} \mathrm{O}_{4}$ at $300 \mathrm{~K}$ (Figure 3a). Zero-field cooling (ZFC) 

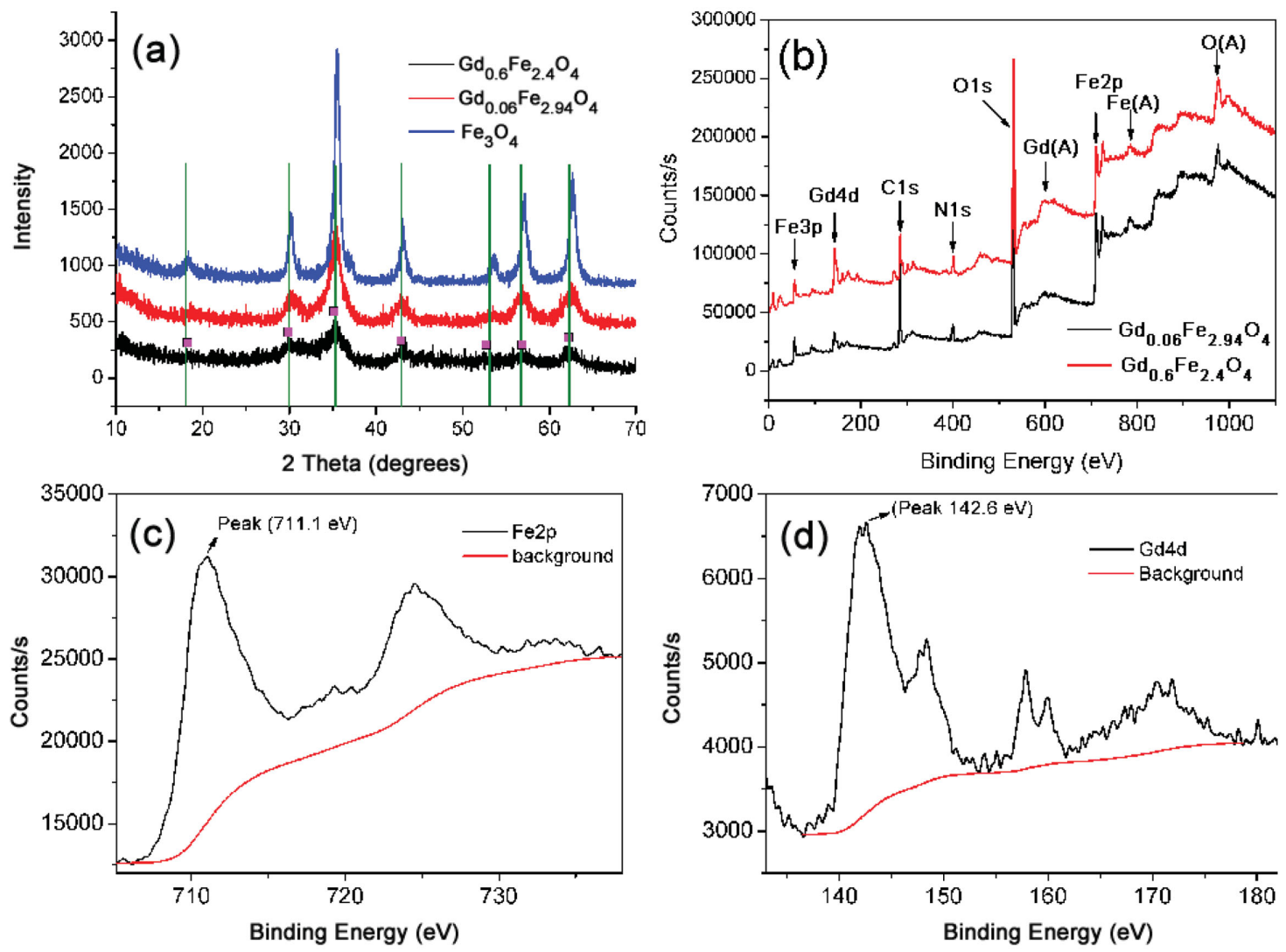

Figure 2. a) XRD patterns of $\mathrm{Fe}_{3} \mathrm{O}_{4}, \mathrm{Gd}_{0.06} \mathrm{Fe}_{2.96} \mathrm{O}_{4}$, and $\mathrm{Gd}_{0.6} \mathrm{Fe}_{2.4} \mathrm{O}_{4}$; b) full XPS spectra of $\mathrm{Gd}_{0.06} \mathrm{Fe}_{2.96} \mathrm{O}_{4}$ and $\mathrm{Gd}_{0.6} \mathrm{Fe}_{2.4} \mathrm{O}_{4}$; c) XPS Fe2p peaks and d) $\mathrm{Gd} 4 \mathrm{~d}$ peaks of $\mathrm{Gd}_{0.6} \mathrm{Fe}_{2.4} \mathrm{O}_{4}$.

and field cooling (FC) measurements gave the blocking temperature $\left(T_{\mathrm{B}}\right)$ of GION under an applied magnetic field 100 Oe (Figure 3b-e). It could be seen that blocking temperature decreased with increasing Gd content in GION, indicating that superparamagnetism of GION could be adjusted by controlling $\mathrm{Fe} / \mathrm{Gd}$ ratio in GION. However, once $\mathrm{Gd} / \mathrm{Fe}$ ratio exceeded $30 \%$, magnetic property of GION was similar to cubic $\mathrm{Gd}_{2} \mathrm{O}_{3}$ (Figure $3 \mathrm{a}, \mathrm{f}$ ), indicating that the $\mathrm{Gd}-\mathrm{O}-\mathrm{Fe}$ bond changed to a Gd-O-Gd bond due to excess Gd atoms existing in GION. The superparamagnetism of GION ensures the spin order of Gd(III) in the inner location of GION has the same direction under an external magnetic field. As a result, a local magnetic field could be formed, which obviously increases the relaxivity of Gd ions and magnetic field inhomogeneity of the $T_{2}$ contrast agent, and then further enhances the $T_{1}$ or $T_{2}$ contrast effect. ${ }^{[35]}$ Therefore, we hypothesized that suitable $\mathrm{Gd} / \mathrm{Fe}$ ratio in GIONs could show excellent magnetic property, resulting in good $T_{1}-T_{2}$ dual-modal ability. On the basis of the above magnetic results, it can be envisioned that $\mathrm{Gd}_{0.6} \mathrm{Fe}_{2.4} \mathrm{O}_{4}$ might have great potential in $T_{1}$ and $T_{2}$ relaxation enhancements because it showed good superparamagnetism, and suitable saturation Ms and Gd content.

To confirm the above hypothesis, the contrast enhancement ability of GIONs with different $\mathrm{Gd} / \mathrm{Fe}$ ratios was investigated using a 9.4 T MRI scanner. Longitudinal $\left(r_{1}\right)$ and transverse relaxivity $\left(r_{2}\right)$ values of GION were calculated through the ratio of $1 / T_{i}$ ( $i=1$ and 2 ) to metal ions concentration for evaluating contrast agent performance. With increased Gd contents in GION, we observed increases in both $r_{1}$ and $r_{2}$ values. GIONs with $1 / 4$ of $\mathrm{Gd} / \mathrm{Fe}$ ratio $\left(\mathrm{Gd}_{0.6} \mathrm{Fe}_{2.4} \mathrm{O}_{4}\right)$ displayed the highest $r_{1}$ and $r_{2}$ values at $7.87 \times 10^{-3}$ and $182.7 \times 10^{-3} \mathrm{M}^{-1} \mathrm{~s}^{-1}$, respectively, which were both much higher than those of the same size of $\mathrm{Gd}_{2} \mathrm{O}_{3}$ and $\mathrm{Fe}_{3} \mathrm{O}_{4} \cdot{ }^{[36,37]}$ However, once the ratio of $\mathrm{Gd} /$ Fe exceeded $1 / 4$ to $1 / 2$, the $r_{1}$ and $r_{2}$ values of GION decreased rapidly to $3.02 \times 10^{-3} \mathrm{M}^{-1} \mathrm{~s}^{-1}$ and $1.6 \times 10^{-3} \mathrm{M}^{-1} \mathrm{~s}^{-1}$, respectively. The $r_{1}$ and $r_{2}$ values of different $\mathrm{Gd} / \mathrm{Fe}$ ratios were also summarized in Figure S4 (Supporting Information), indicating that GIONs with $1 / 4$ of $\mathrm{Gd} / \mathrm{Fe}$ ratio possessed the best MRI performance. Therefore, optimal Gd/Fe ratio in GION could be confirmed at $1 / 4$, indicating that GION with $1 / 4$ of $\mathrm{Gd} / \mathrm{Fe}$ ratio can act as both excellent negative and positive contrast agents by a synergistically enhanced contrast effects. In alignment with our hypothesis, these results demonstrated the synergistic enhancement of $r_{1}$ and $r_{2}$ relaxivity in GION.

\subsection{Drug Loading and Release Behavior}

It has been established that the microenvironment of tumor cells is weakly acidity, such as tumor and inflammatory tissues ( $\mathrm{pH}$ 6.5), corresponding endosomes ( $\mathrm{pH}$ 5.5-6.0), and lysosomes (pH 4.5-5.0). ${ }^{[38,39]}$ Therefore, it will undoubtedly 

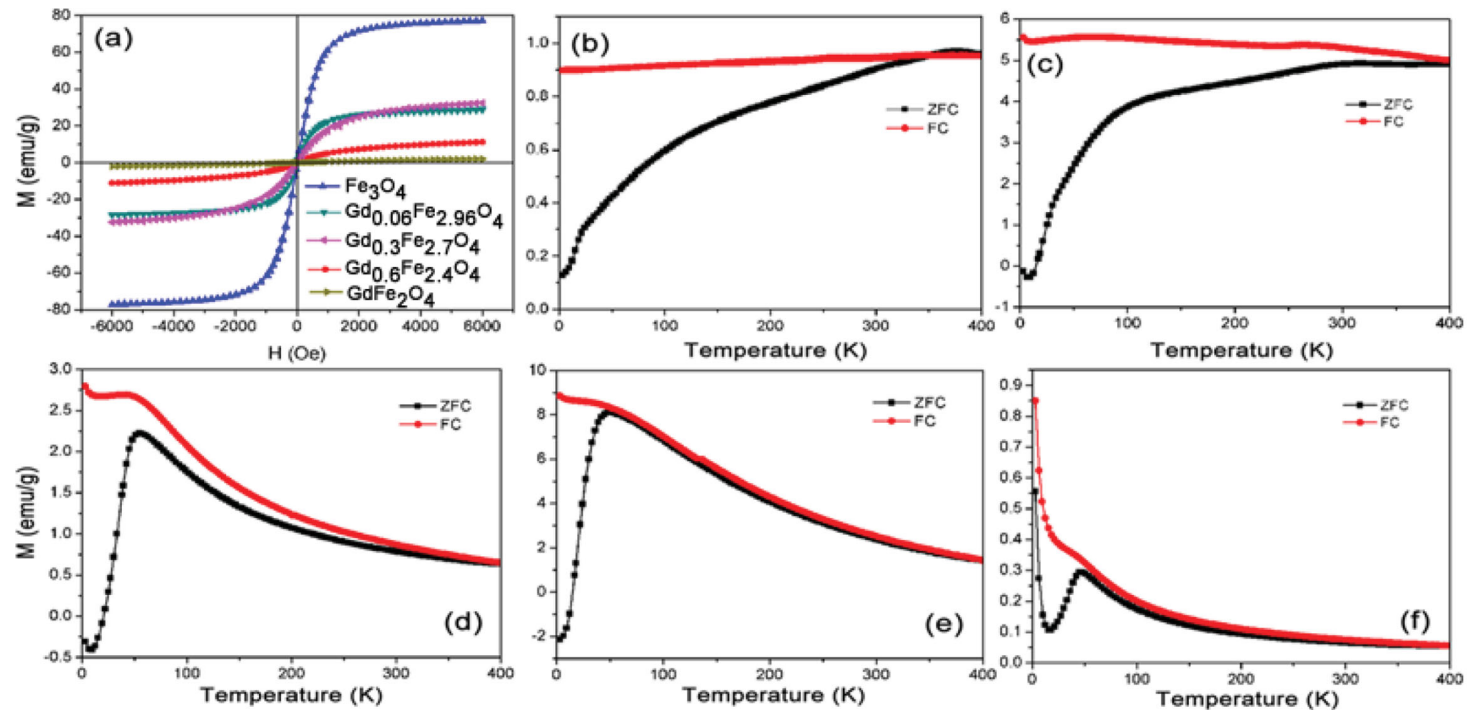

Figure 3. a) Hysteresis loops of samples at $300 \mathrm{~K}$; the temperature dependence of $\mathrm{ZFC}$ and $\mathrm{FC}$ magnetization curves for b) $\left.\mathrm{Fe}_{3} \mathrm{O}_{4}, \mathrm{c}\right) \mathrm{Cd}_{0.06} \mathrm{Fe}_{2.94} \mathrm{O}_{4}$, d) $\mathrm{Cd}_{0.3} \mathrm{Fe}_{2.7} \mathrm{O}_{4}$, e) $\mathrm{Gd}_{0.6} \mathrm{Fe}_{2.4} \mathrm{O}_{4}$, and f) $\mathrm{GdFe}_{2} \mathrm{O}_{4}$.

help to kill tumor cells much more effectively if GION loaded with drugs possess $\mathrm{pH}$-dependent release behavior. As shown in Scheme 1, DOX was loaded onto GION by a $\mathrm{pH}$-sensitive hydrazone bond, and Fourier transform infrared (FTIR) spectra were used to monitor the modification process (Figure S5, Supporting Information). The peak at $586 \mathrm{~cm}^{-1}$ in all curves was attributed to the typical $\mathrm{Fe}-\mathrm{O}$ stretching vibration. In the curve of GION, the peaks at 1075, 1634, 2938, and $2853 \mathrm{~cm}^{-1}$ were respectively ascribed to $\mathrm{C}-\mathrm{N}$ stretching vibration, $\mathrm{N}-\mathrm{H}$ bending vibration, and $\mathrm{C}-\mathrm{H}$ stretching vibration, indicating that GION contained plenty of PEI chains. After the hydrazide bonds were formed on the GION, new peaks at 1648 and $3314 \mathrm{~cm}^{-1}$ appeared in the GION-hydrazide curve, which was assigned to the characteristic bond of linkage in $-\mathrm{CH}_{2} \mathrm{CH}_{2} \mathrm{CONHNH}_{2}$. In addition, in the curve of GION-DOX, the characteristic peaks at 1540,1460 , and $1410 \mathrm{~cm}^{-1}$ corresponded to the skeleton vibration of the benzene ring in DOX, indicating that the DOX was successfully conjugated to GION. It was noteworthy that the peak at $1715 \mathrm{~cm}^{-1}$ belonging to $\mathrm{C}=\mathrm{O}$ stretching vibration of free DOX was absent, thus indicating that DOX was covalently bound with GION. Interestingly, in the curve of FA-GION-DOX, the characteristic peak of $\mathrm{C}=\mathrm{O}$ stretching vibration reappeared at $1687 \mathrm{~cm}^{-1}$, indicating FA was linked to GION-DOX by amide bond. The loading capacity of DOX conjugated into GION was calculated to be $\approx 9.6 \mathrm{wt} \%$ by UV-vis analysis.

Subsequently, we investigated the release behavior of DOX from GION-DOX using UV-vis spectra $(490 \mathrm{~nm})$ in phosphate buffers at neutral and weakly acidic $\mathrm{pH}$. The release curves of DOX were shown in Figure 4a. The release amount of DOX molecules was negligible for GION-DOX at $\mathrm{pH}$ 7.4, indicating that the hydrazone bond linking the GION and DOX was very stable at neutral condition. However, a marked increase in DOX concentration of the supernatant was observed at $\mathrm{pH}$ 5.5, indicating that DOX molecules were released efficiently from GION-DOX under the weakly acidic conditions. Additionally, the release rate of DOX was faster with decreasing $\mathrm{pH}$ value, displaying typical $\mathrm{pH}$-dependent release behavior.

\subsection{Cell Viability, Uptake, and Localization}

In order to evaluate the application of GION for cancer treatment and diagnosis, HeLa cells incubated with free DOX, GION, GION-DOX, and FA-GION-DOX were assessed through a Cell Counting Kit-8 (CCK-8) assay. GIONs did not result in a significant decrease in cell viability at a wide range of concentrations. This was an indication that the toxicity of GIONs was negligible (Figure 4d). However, GION-DOX exhibited a dose-dependent cytotoxic effect, which was similar to that observed with equivalent doses of free DOX when the concentration of DOX was above $12 \mu \mathrm{g} \mathrm{mL}^{-1}$ (Figure 4e). This is crucial that GION-DOX showed low cytotoxicity when the concentration of DOX was less than $12 \mu \mathrm{g} \mathrm{mL}^{-1}$. This could be explained that low dose of GION-DOX cannot be internalized by HeLa cells, resulting in the low release amount of DOX. Compared with GION-DOX, FA-GION-DOX displayed a significant reduction in cell viability after a $24 \mathrm{~h}$ incubation period, indicating that folic acid was beneficial for the uptake of FA-GION-DOX on HeLa cells and effect of DOX on the cancer cells. Flow cytometry results showed obvious lower cellular uptake of GION-DOX and FA-GION-DOX in the presence of folic acid than that of FA-GION-DOX within $24 \mathrm{~h}$ of incubation (Figure 4f).

To further investigate the effects of nanomaterials on intracellular localization of DOX, their intracellular localization in the living cells was observed by confocal microscopy (Figure 5). For HeLa cells treated with free DOX, red fluorescence was visible inside cells, suggesting fast diffusion and internalization after $4 \mathrm{~h}$ of incubation. Meanwhile, the GION-DOX exhibits weak green fluorescence in cytomembrane and negligible red fluorescence in cytoplasm, which suggested that GION-DOX cannot 

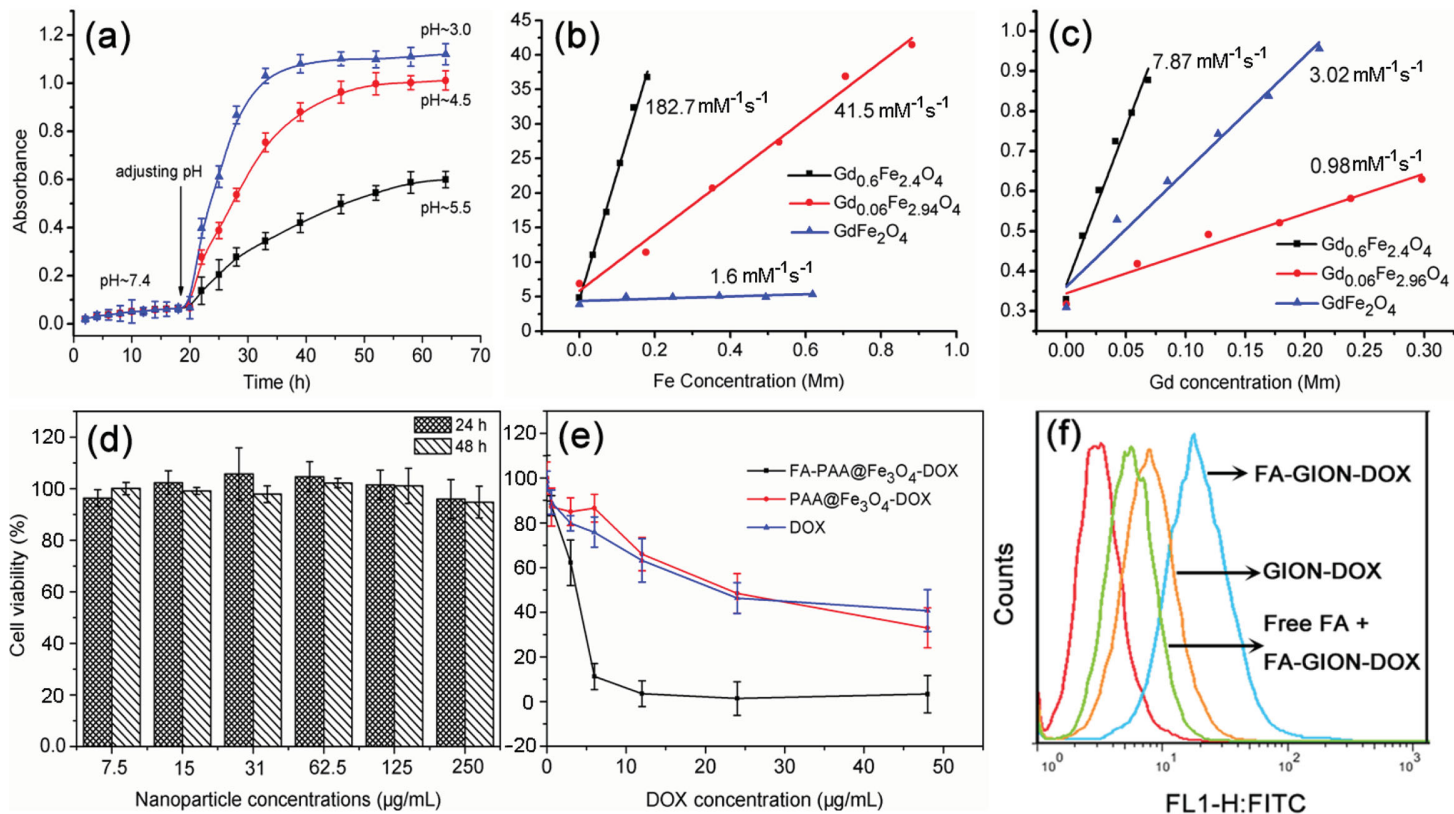

Figure 4. a) Release behavior of FA-GION-DOX under different $\mathrm{pH}$ conditions; b) the transverse relaxation rate of samples through the ratio of $1 / T_{2}$ to Fe concentration; c) the longitudinal relaxation rate of samples through the ratio of $1 / T_{1}$ to $\mathrm{Gd}$ concentration; d) CCK-8 assay for HeLa cells viabilities after incubation with different concentrations of FA-GION; e) viabilities of HeLa cells treated with free DOX, GION-DOX, FA-GION-DOX at different concentrations for $12 \mathrm{~h}$; f) flow cytometry analysis of HeLa cells incubated with FITC labeled different samples for $2 \mathrm{~h}$. The red line represents the negative control sample.

be internalized by HeLa cells, resulting in no DOX release from GION-DOX. However, all of the HeLa cells showed a bright fluorescence in green and red light, which indicated that FA-GION-DOX exhibited efficient intracellular delivery in tumor cells with colocalization in lysosome and entering into the nucleus. These results confirmed that FA-GION-DOX can effectively deliver the drugs into living cells and possess a $\mathrm{pH}$ dependent drug release profile within cancer cells.

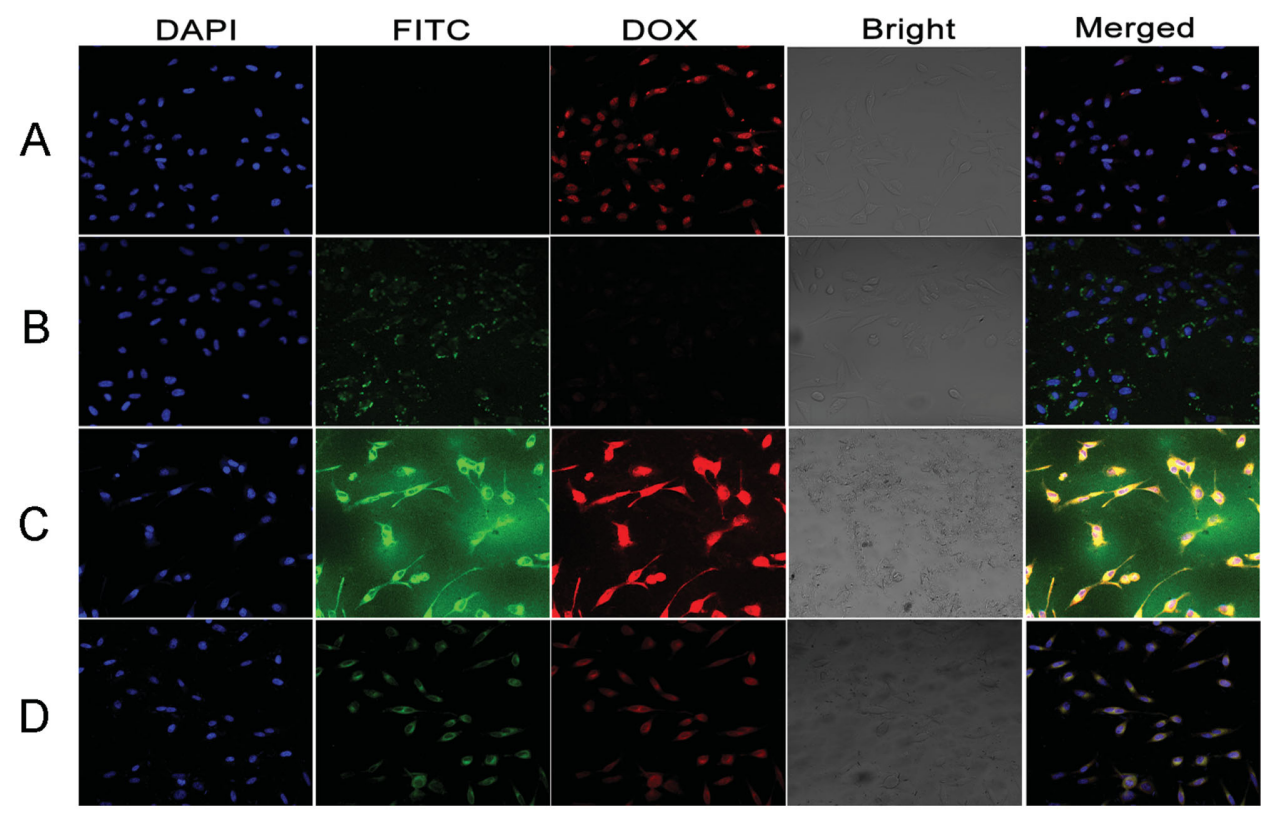

Figure 5. Confocal laser scanning microscopy (CLSM) observations of HeLa cells incubated with A) DOX, B) FITC labeled GION-DOX, and FAGION-DOX in the C) absence and D) presence of $5 \times 10^{-3} \mathrm{M}$ free folic acid. For each panel, the images from left to right show cell nuclei stained by DAPI (blue; DAPI: 4',6-diamidino-2-phenylindole), FITC fluorescence in cells (green), DOX fluorescence in cells (red), bright field, and the merged one of the left four images. All images share the same scale bar. 


\subsection{Antitumor Effect in a Subcutaneous Human Cervical Cancer Xenograft Model}

The in vivo therapeutic efficacy of free DOX, GION-DOX, and FA-GION-DOX for cervical cancer was evaluated in BALB/C nude mice (Figure 6a). The tumor growth rates of mice treated with physiological saline (control) showed rapid tumor growth, indicating that physiological saline alone had no effect on tumor growth. In contrast, the groups injected with the free DOX or GION-DOX had greatly delayed and inhibited tumor growth. After $38 \mathrm{~d}$, free DOX and GION-DOX suppressed tumor volumes by $62.5 \%$ and $37.5 \%$, respectively, compared with the control group. However, tumor volume appeared as explosive growth for injecting free DOX or GION-DOX to mice, which was ascribed to resistant drug of cancer cells. ${ }^{40,41]}$ In contrast, FA-GION-DOX displayed a significant inhibitory effect in tumor size, which may be mainly due to the sustained DOX release in vivo in the tumor tissue after particle accumulation via targeting effect of folic acid. Images of Figure $6 \mathrm{~b}$ were consistent with Figure 6a, showing that FA-GION-DOX possessed a stronger inhibitory effect on tumor growth.

We further investigated the biodistribution of GION-DOX and FA-GION-DOX in vivo. After injection by caudal vein about $30 \mathrm{~min}$, the mice were sacrificed and the tumors and major organs (e.g., lymph, liver, kidney, and spleen) were collected. The samples were then evaluated through measurement of $\mathrm{Gd}^{3+}$ content using ICP-MS, and the data are presented as the accumulation of GION per mass unit of an organ (Figure $6 \mathrm{c}$ ). The amount of FA-GION-DOX in the tumor was 3-fold higher than that of GION-DOX, which suggested that folic acid displayed good targeting in vivo.

In addition, the biodistribution data showed that considerable amounts of GIONs and FA-GIONs accumulated in the liver and spleen due to the reticuloendothelial system. We suppose that FA-GIONs tend to accumulate in tissues with a high density of folate receptors and could be excreted from the body mainly through hepatobiliary clearance. ${ }^{[42]}$ The data suggest that FA-GION-DOX can selectively recognize the tumor tissues among different tissues as a result of the hydrolyzation of hydrazone bonds induced by the acidic tumor microenvironment. Compared to traditional chemotherapy, nanoparticle-mediated drug delivery reduces the risks associated with systemic distribution of the drug mitigating unwanted side effects. ${ }^{[43]}$ Side effects of free DOX, GION-DOX, and FA-GION-DOX were evaluated through changes in weight of the mice. As shown in Figure 6d, the weight of mice did not increase with injection of free DOX, suggesting that DOX influenced the normal growth of mice as a result of damage caused by free DOX on normal tissues. ${ }^{[44]}$ However, the weight of mice has the normal growth from 18 to $25 \mathrm{~g}$ by injecting GION-DOX. Importantly, the treatment of FA-GION-DOX caused a significant increase in the weight of mice because the cancer was well controlled. These results suggest that FA-GION-DOX can reduce the side effects of DOX chemotherapy and enhance the survival rate of cancer patients.

Hematoxylin and eosin (H\&E) staining results of hypodermatic tumors (Figure 7) showed numerous necrotic areas and highly malignant hyperchromatin tumor cells in the

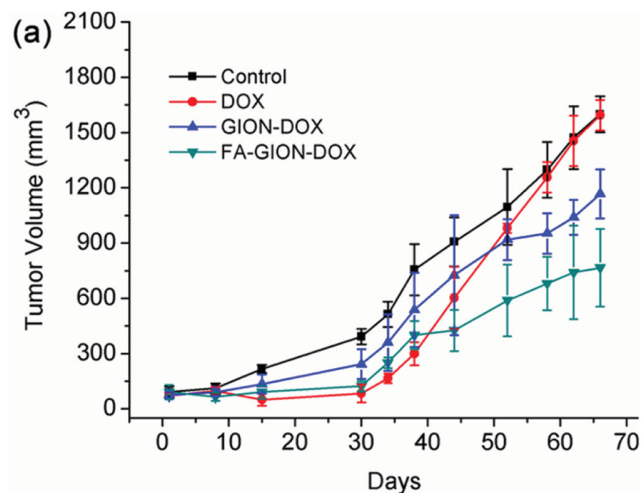

(b)
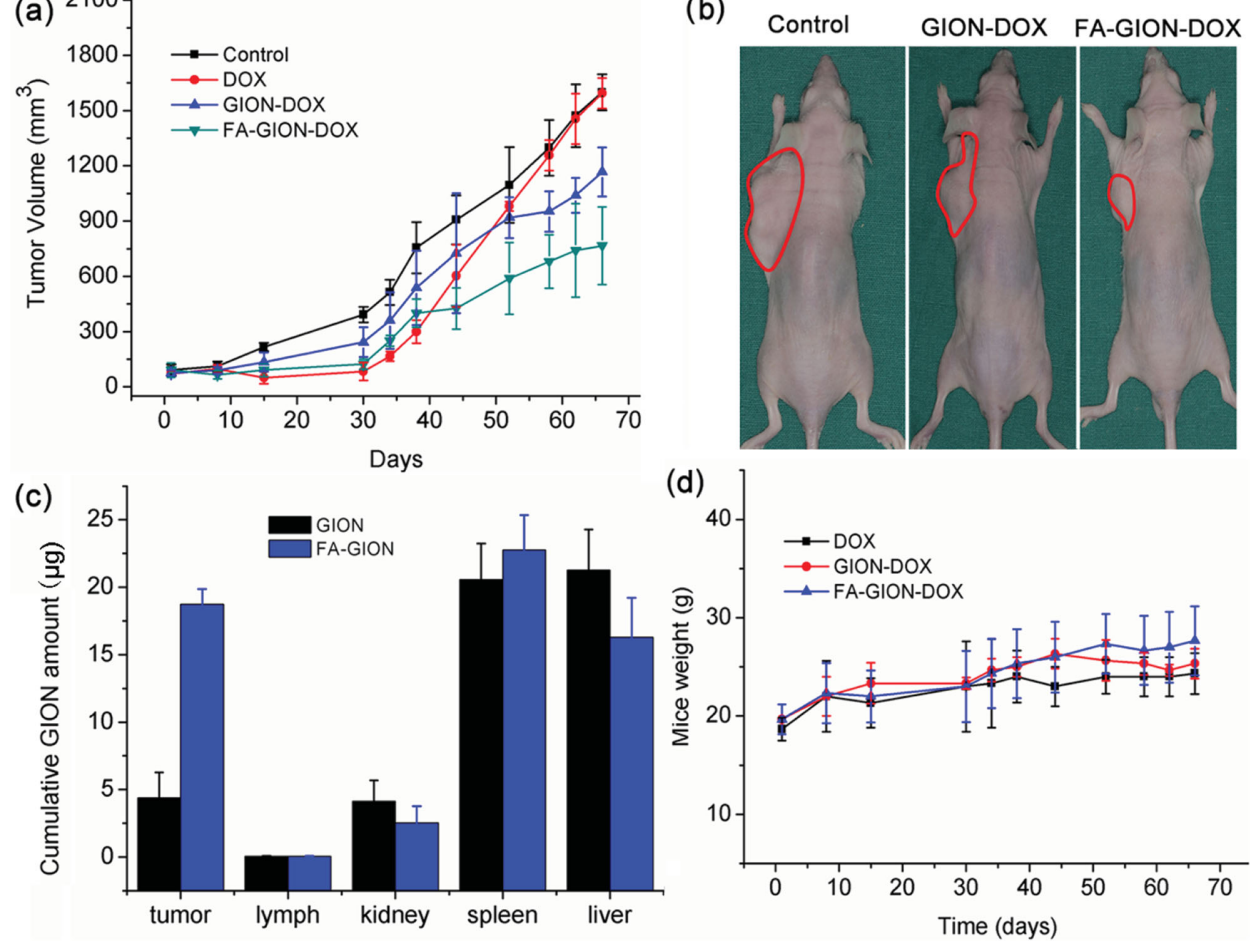

Figure 6. a) Tumor volume changes of saline-treated mice compared to mice treated with DOX, GION-DOX, and FA-GION-DOX over 2 months; b) photos of mice treated with different samples; c) in vivo biodistribution of GION-DOX and FA-GION-DOX in nude mice of bearing cancer after the intravenous injection ( $\mathrm{GION}, 300 \mu \mathrm{g})$; d) weight changes of mice treated with different samples. 
(1)
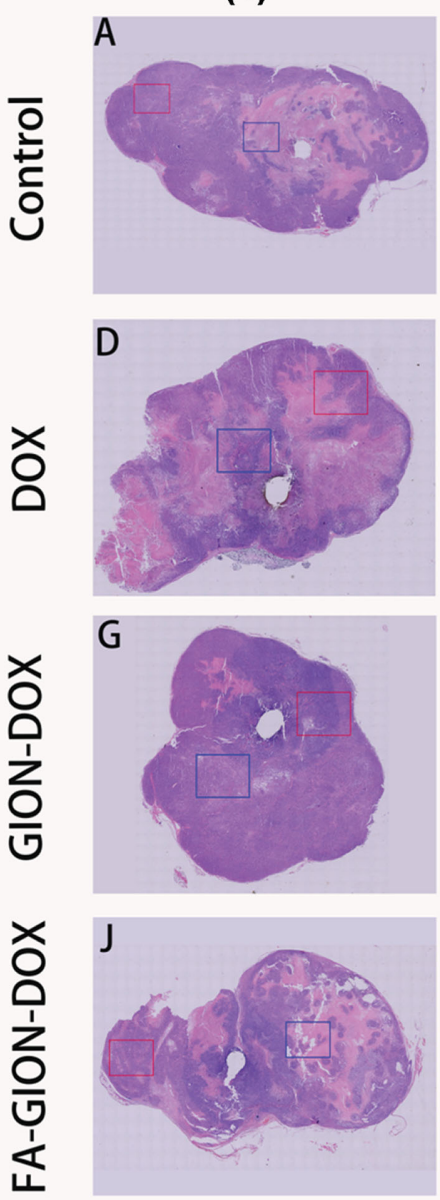

(2)
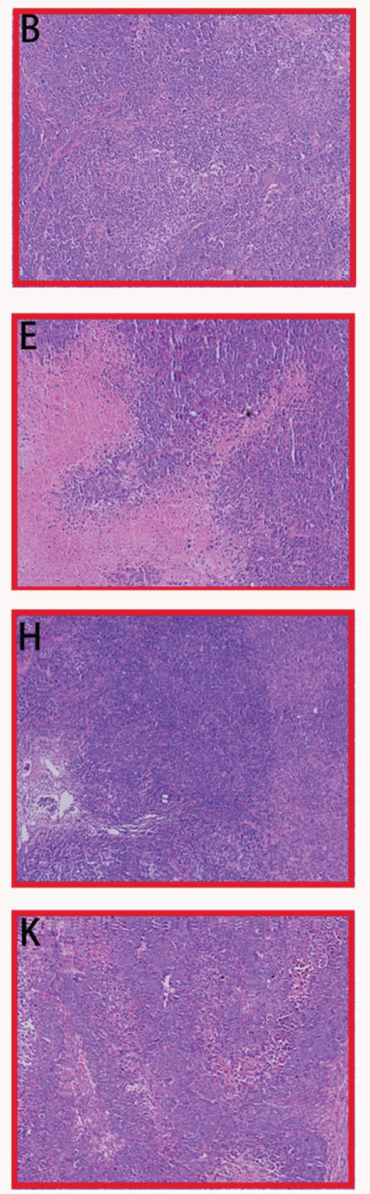

(3)
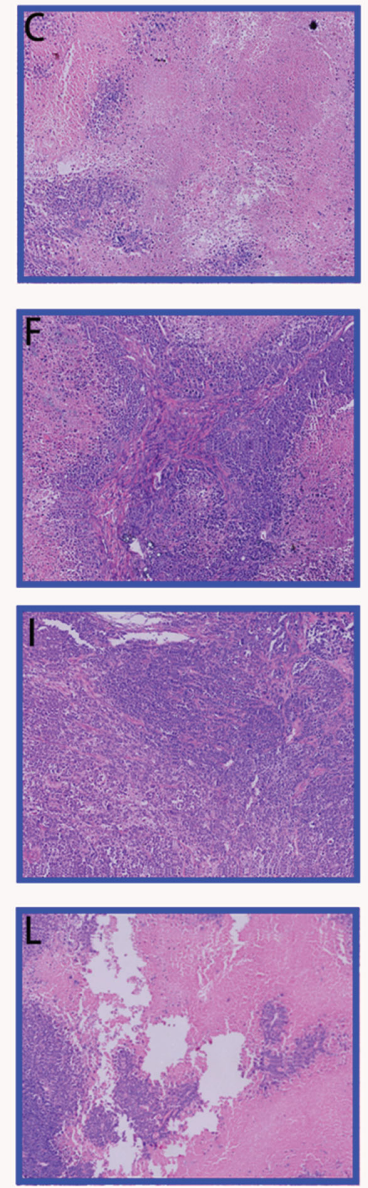

Figure 7. 1) Photomicrographs of HeLa cell xenograft tumors with H\&E staining. 2) Hyperchromatin cell density of tumor tissues in boundary: $B>E>H>K$. (3) Necrotic areas of tumor tissues in core: $C<F<I<L$.

slides. Nuclear atypia could also be observed inside the cells of tumors. Tumor cell density and quantity of atypical nuclei were estimated approximately by experienced pathologists under optical microscope with $200 \times$ magnification. The pathologists selected the boundary and core tissues of tumors in every slides, respectively, and gave analysis as follows: (1) necrotic areas in slides of experimental groups (FA-GION-DOX) were more than control groups; (2) the density of hyperchromatin tumor cells of experimental group was smaller than that of control group while the quantity of atypical nuclei was larger than that of control group.

\subsection{Monitoring Targeted Delivery of FA-GION-DOX by Noninvasive MRI}

To observe if the systemic delivery of FA-GION-DOX leads to the specific accumulation of nanoparticles in tumors, the tumor-bearing mice were observed by MRI, following the FAGION-DOX treatment. $T_{1}$ and $T_{2}$ images at transverse planes of region of interest tumors were acquired at preinjection and
$15,30,60$, and 120 min postinjection of FA-GION-DOX or GION-DOX with a dose of $2.0 \mathrm{mg} \mathrm{kg}^{-1}$ mouse body weight through tail vein. We found that $T_{2}$-weighted images of tumor in mice treated with FA-GION-DOX appeared significantly darken at $30 \mathrm{~min}$, which suggested that FA-GION-DOX nanoparticles were accumulated in tumor area (Figure 8a). Moreover, the MRI signal intensity of $T_{2}$-weighted images decreased $33 \%$ after the tumors of mice were injected by FA-GION-DOX for $30 \mathrm{~min}$, compared to the signal level obtained before treatment (Figure 8b). Following this, the MRI signal change dropped at $120 \mathrm{~min}$ may be due to excretion of FA-GION-DOX by metabolism pathway. However, $T_{2}$-weighted MRI showed no significant signal decrease in tumors of the mice treated with nontargeted GION-DOX. Correspondingly, $T_{1}$ signal changes were demonstrated at the same time and showed similar to $T_{2}$ signal results. As shown in Figure 8c, $T_{1}$-weighted MR images exhibited a significantly brighter signal in the tumor of mouse with FAGION-DOX at 15 min postinjection. The analysis of MRI signal changes in the tumor region indicated that the biggest intensity change, of $\approx 74 \%$ in $T_{1}$ imaging, occurred at 
(a)

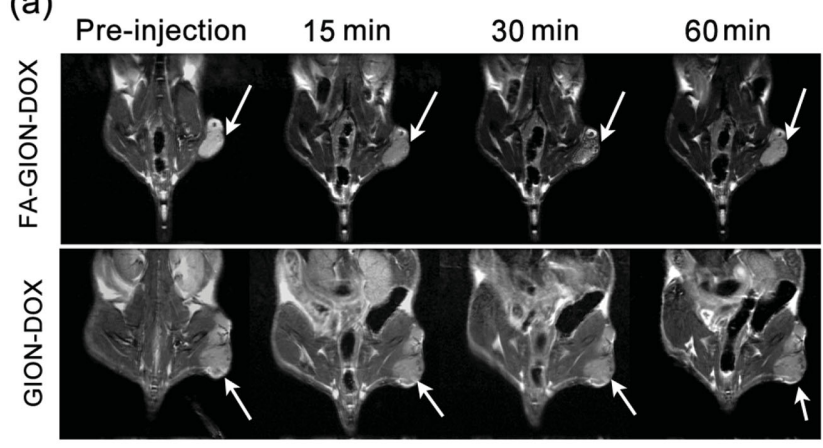

(c)

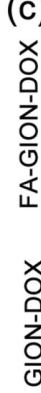

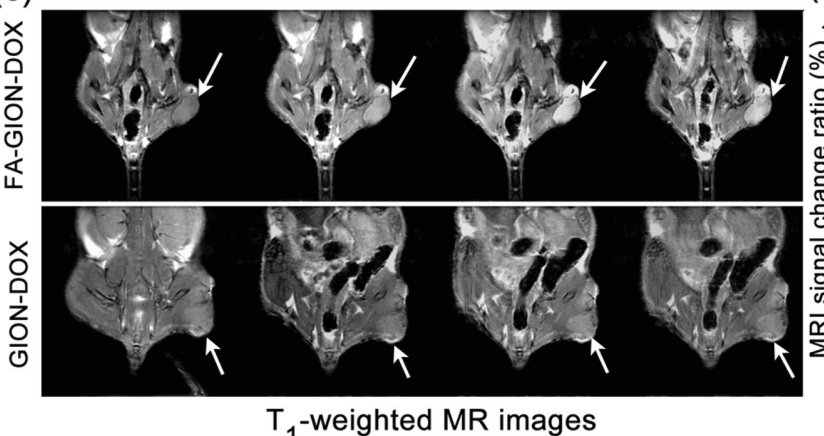

(b)

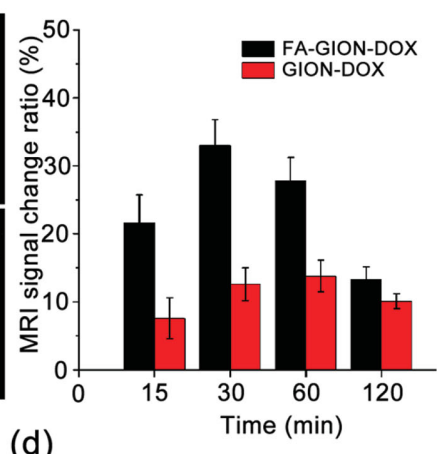

(d)

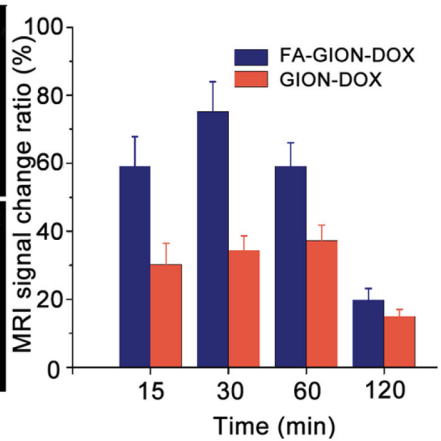

Figure 8. Delivery of FA-GION-DOX in tumor by $T_{1}-T_{2}$ dual-modal MRI monitoring. a) $T_{2}$ - and c) $T_{1}$-weighted MR images (9.4 T) of mice acquired at preinjection and 15, 30, and 60 min postinjection of FA-GION-DOX and GION-DOX with a dose of $2.0 \mathrm{mg}$ (GION) $\mathrm{kg}^{-1}$ (mouse body weight). MRI signal changes in tumor of c) $T_{2}$ - and d) $T_{1}$-weighted images at transverse planes ( $n=3$, data represent mean \pm standard deviation), showing that maximal signal changes in tumor were achieved at postinjection about 30 min. Tumor marked by a white arrow.

60 min postinjection (Figure 8d). However, tumors treated with GION-DOX displayed a weak brighter area in $T_{1}$ weighted images and only $31 \%$ enhancement in MRI signal change. These results suggest that FA-GION-DOX has potential to serve as a targeted $T_{1}-T_{2}$ dual-model MRI contrast agent for tumor imaging, which may be helpful for accurate diagnosis and treatment of cancer.

\section{Conclusion}

In summary, we introduced an efficient strategy to develop a high-performance MRI contrast agent and targeted drug delivery system, FA-GION-DOX, via modifying surface structure and nanobiointerface. GION displayed a significant contrast enhancement in $T_{1}$ and $T_{2}$ imaging, which could be attributed to the synergetic effect of $\mathrm{Gd}$ and Fe atoms. Meanwhile, targeted FA-GION-DOX resulted in pH-dependent controlled release of the therapeutic agent. This FA-GION-DOX nanosystem not only enables intracellular drug release, but also allows the detection of the nanodrug accumulation in tumors by $T_{1}-T_{2}$ dual-model imaging. Our results demonstrated that systemic delivery of FA-GION-DOX significantly inhibited tumor growth in a human cervical cancer xenograft model. We further showed that targeted delivery of the FA-GION-DOX and the presence of the nanodrug could be detected in the tumor through noninvasive means, using both $T_{2}$-weighted and $T_{1}$-weighted MRI. Therefore, FA-GION-DOX developed in this study is a promising drug delivery system for the targeted and image-guided therapy of cervical cancer.

\section{Experimental Section}

Materials: All chemical reagents were used as received without further purification. Ethylene glycol, triethanolamine (TEA), dimethylsulfoxide, polyethylene glycol $\left(M_{\mathrm{w}}: 400\right)$ were purchased from Sinopharm Co. (Shanghai, China). DOX, PEI, N-hydroxysuccinimide (NHS), N-ethyl-N'(3-dimethylaminopropyl) carbodiimide (EDC), $\mathrm{Gd}(\mathrm{acac})_{3}$, and $\mathrm{Fe}(\mathrm{acac})_{3}$ were received from Aladdin Co. (Shanghai, China). Hoechst 33342, trypan blue, propidium iodide were purchased from Sigma-Aldrich. Co. (USA). The CCK-8 was obtained from Dojindo (Japan), and an Annexin V-FITC Apoptosis Detection Kit was purchased from BD Biosciences (San Jose, CA, USA). All other analytical grade chemicals were obtained from Sinopharm Group Chemical Reagent Co., Ltd (Shanghai, China).

Synthesis of GION: The various ratios of iron acetylacetonate and gadolinium acetylacetonate complexes were dissolved in a mixed solution of ethylene glycol $(15 \mathrm{~mL})$ and polyethylene glycol $(35 \mathrm{~mL})$ for $30 \mathrm{~min}$ at $80^{\circ} \mathrm{C}$. Next, PEl was added in the solution to form a brown turbid solution for $30 \mathrm{~min}$. Afterward, TEA $(3 \mathrm{~mL})$ was added under stirring to form a homogeneous and transparent solution. This solution was then transferred to an autoclave and kept at $230{ }^{\circ} \mathrm{C}$ for $2 \mathrm{~d}$. Finally, the black product was collected and washed at least three times with distilled water and alcohol.

Preparation of FA-GION-DOX: (1) GIONs contained with amino groups were dispersed in distilled DMF for $2 \mathrm{~h}$ at $60^{\circ} \mathrm{C}$. Subsequently, triethylamine was added in the resulting solution under nitrogen. After $30 \mathrm{~min}$, DMF containing acrylic ester was added to the above solution under nitrogen, and allowed to react for $12 \mathrm{~h}$ to form GIONvinyl. Subsequently, hydrazine was further added in the mixed solution 
contained GION-vinyl for $4 \mathrm{~h}$. After that, the active ester of folic acid prepared as reported previously ${ }^{[45]}$ was added into the resulting solution for $6 \mathrm{~h}$, and then folic acid could link to GION-hydrazine through conjugating the rest amine groups on the surface of GION. The product (FA-GION-hydrazine) was collected by external magnets and washed using ethanol. (2) DOX was conjugated to FA-GION-hydrazine through formation of hydrazone bonds. Commonly, DOX and acetic acid were added to methanol solution of GION-hydrazine and reacted for $2 \mathrm{~d}$ under nitrogen condition at room temperature. The product (FA-GIONDOX) was collected by external magnets and washed with ethanol until the supernatant was clear, with no color being visibly detectable.

Release Kinetics of FA-GION-DOX: FA-GION-DOX (10 mg) was first dispersed into simulated body fluid $(\mathrm{pH} 7.4,10 \mathrm{~mL})$. Then, these solutions were poured into dialysis bags. While stirring, the dialysis bags were rapidly immersed in the same buffer $(100 \mathrm{~mL})$ at room temperature for $20 \mathrm{~h}$. Subsequently, the $\mathrm{pH}$ values of medium were adjusted to 5.5, 4.5 , or 3.0 by adding $\mathrm{HCl}$ solution $(0.05 \mathrm{M})$. Medium was collected at defined time intervals and the amount of released DOX was determined using a UV-vis spectrophotometer at a wavelength of $488 \mathrm{~nm}$.

MR Experiment: The capability of nanoplatforms to influence $T_{1}$ or $T_{2}$ relaxation time was studied using a $9.4 \mathrm{~T}, 8.9 \mathrm{~cm}$ wide bore, actively screened, transverse bore MR spectrometer (Bruker Biospoin $\mathrm{GmbH}$, Germany). The longitudinal $\left(T_{1}\right)$ and transverse $\left(T_{2}\right)$ relaxation rates were measured using the inversion recovery method and a multi-echo spin echo sequence, respectively. The relaxivities $\left(r_{1}\right.$ and $\left.r_{2}\right)$ were determined by a linear fit of the inverse relaxation time as a function of metal ions concentration, which was determined using ICP-MS (ICP6300, Thermo Fisher Scientific Co., USA).

Cell Culture and Cytotoxicity Assay: HeLa cells were plated into 96-well plates at $1 \times 10^{4}$ cells per well and maintained in dulbecco's modified eagle medium (DMEM) supplemented with 10\% fetal bovine serum and $1 \%$ penicillin/streptomycin at $37{ }^{\circ} \mathrm{C}$ in a humidified atmosphere of $5 \%$ $\mathrm{CO}_{2}$. After being cultured for $24 \mathrm{~h}$, HeLa cells were treated with various concentrations of DOX, GION-DOX, and FA-GION-DOX, and allowed to further incubate for an additional $24 \mathrm{~h}$. In addition, HeLa cells were treated with different concentrations of GION for $48 \mathrm{~h}$. After incubation with various samples, the culture media were removed and cells were washed with phosphate buffer solution (PBS). The number of viable cells was determined using a CCK-8 (BestBio, China). Absorbance was measured at $450 \mathrm{~nm}$ using a microplate reader (ChroMate 4300, USA). All tests were carried out at least in triplicate.

Fluorescein isothiocyanate (FITC) Accumulation Assay: FITC labeled GION, FA-GION were fabricated by previously described methods. HeLa cells seeded in $60 \mathrm{~mm}$ plates $\left(5 \times 10^{5}\right.$ cells per dish) were treated with exogenous free folic acid $\left(5 \times 10^{-3} \mathrm{M}\right)$ for $2 \mathrm{~h}$ at $37^{\circ} \mathrm{C}$ followed by FITCFA-GION $\left(40 \mu \mathrm{g} \mathrm{mL}^{-1}\right)$ for $4 \mathrm{~h}$. HeLa cells were also incubated with GION-FITC or FA-GION-FITC at the same concentration $\left(40 \mu \mathrm{g} \mathrm{mL}^{-1}\right)$. After $4 \mathrm{~h}$, trypan blue $(0.4 \%, 1 \mathrm{~mL})$ was used to quench the extracellular fluorescence for $2 \mathrm{~min}$. Cells were next trypsinized, washed three times with ice-cold PBS, resuspended in PBS $(500 \mu \mathrm{L})$, and measured by flow cytometry (BD FACScan flow cytometer, BD Biosciences).

Confocal Microscopy: HeLa cells were seeded on $15 \mathrm{~mm}$ glass coverslips placed in 12-well plates $\left(8 \times 10^{4}\right.$ cells per well) and incubated with exogenous free folic acid $\left(5 \times 10^{-3} \mathrm{M}\right)$ and after $2 \mathrm{~h}$ the cells were further incubated with FA-GION-FITC $\left(40 \mu \mathrm{g} \mathrm{mL}^{-1}\right)$ for 1,2 , and $4 \mathrm{~h}$. Additionally, HeLa cells were also incubated with FITC-GION or FITC-FA-GION at the same concentration for 1,2 , and $4 \mathrm{~h}$, followed by nuclei staining using the Hoechst Dye (Hoechst 33342). Cells were washed three times using PBS and fixed in freshly prepared $4 \%$ paraformaldehyde. Slides were coverslipped and visualized using confocal microscope (Zeiss LSM710 NLO, Germany).

Human Cervical Tumor Xenograft Mouse Model: The cervical cancer model was established through direct subcutaneous injection of $5 \times 10^{7} \mathrm{HeLa}$ cells into the left axilla of nude mice. Three to four weeks following injection of cells, cervical tumors of $100 \mathrm{~mm}^{3}$ in volume were apparent. The mice used for the experiment were treated in accordance with the Ethics Committee Guidelines in University of Science and Technology of China. Animals were randomized into four experimental groups of five or six mice as follows: control, free DOX, nontargeted GION-DOX, and FA-GION-DOX. The mice were treated via tail vein injections at $4 \mathrm{~d}$ intervals. On each treatment $d$, all groups except for the control group were administered an equivalent dose of $2 \mathrm{mg} \mathrm{kg}^{-1}$ of body weight. Control mice were injected saline buffer. The animal study was performed three times under the same conditions, and the results of three separate experiments were combined to analyze values. The volume $(V)$ of tumor was calculated as $V=a \times b^{2} / 2$, where " $a$ " and " $b$ " were the longest and shortest diameters of the tumor, respectively. The body weights of mice were measured and recorded twice a week.

Biodistribution of FA-GION-DOX In Vivo: The biodistribution analysis was performed $30 \mathrm{~min}$ after the injection of the GION-DOX and FA-GION-DOX $\left(2 \mathrm{mg} \mathrm{kg}^{-1}\right)$. The dissected organs were weighed, homogenized, and then treated with scintillation mixtures. A volume of $60 \%$ nitric acid was added to each sample and the tissues were placed on water bath for $24 \mathrm{~h}$ at $60^{\circ} \mathrm{C}$, after which the solutions were centrifuged at $13000 \mathrm{rpm}$ for $10 \mathrm{~min}$ and the supernatant was collected and then subjected to ICP-MS. The GION-DOX and FA-GION-DOX distribution in different organs was calculated as the percentage of the injected dose per gram of tissue.

Pathological Analysis of Tumor Tissue: The tumor tissues were sectioned into two halves and measured. One half was used for quantitative analysis. The other half was fixed in 10\% buffered formalin, and embedded in paraffin for histological analysis using H\&E staining. Tumor cell density, tumor necrosis, mitotic figure count, and nuclear atypia were evaluated with light microscopy using 400x magnification. This analysis was semiquantified by experienced pathologists in a double blind manner. ${ }^{[46,47]}$

In Vivo MRI: HeLa cells $\left(1.0 \times 10^{7}\right.$ cells $)$ were subcutaneously transplanted into $\mathrm{BALB} / \mathrm{C}$ male nude mice. When the tumors grew to $\approx 3 \mathrm{~mm}^{3}$ in volume, GION-DOX and FA-GION-DOX $\left(2 \mathrm{mg} \mathrm{kg}^{-1}\right)$ were intravenously injected into the cervical-cancer-bearing mice. $T_{1}$-weighted images of the mice were acquired using a Litz coil (diameter $100 \mathrm{~mm}$ and length $85 \mathrm{~mm}$ ) on a $9.4 \mathrm{~T}$ MRI scanner (Bruker Biospoin $\mathrm{GmbH}$, Germany). The imaging parameters were as follows: fast spin echo, TR $400.0 \mathrm{~ms}$, TE $10.0 \mathrm{~ms}$, and slice thickness $2.0 \mathrm{~mm}$.

Characterization: The morphologies and element mapping of samples were observed on a TEM (JEM-ARM200F, JEOL Co., Japan). The structure and reaction were monitored using an FTIR spectrometer (Nicolet Co., USA). Particle size distribution measurements were conducted on a dynamic light scattering detector (Malvern, UK).

\section{Supporting Information}

Supporting Information is available from the Wiley Online Library or from the author.

\section{Acknowledgements}

G. Zhang, R. Du, and L. Zhang are co-first authors. All authors acknowledge financial support from the National Natural Science Foundation of China (Nos. 21407151, 31370983, U1232212, and 81201068), the Key Program of Chinese Academy of Sciences (No. KSZD-EW-Z-022-05), and the Science and Technology Service Programs of Chinese Academy of Sciences (Nos. KF)-EW-STS-083 and KF)EW-STS-067), Anhui Province Funds for Distinguished Young Scientists (1508085J08), the Grant of the President Foundation of Hefei Institutes of Physical Science of Chinese Academy of Sciences (No. YZJJ201502), and the Young Top-Notch Talent Support Scheme from Anhui Medical University.

Received: July 12, 2015

Revised: August 5, 2015

Published online: September 9, 2015 
[1] Y. Wang, H. Gu, Adv. Mater. 2015, 27, 576.

[2] N. Insin, J. B. Tracy, H. Lee, J. P. Zimmer, R. M. Westervelt, M. G. Bawendi, ACS Nano 2008, 2, 197.

[3] S. Gai, P. Yang, C. Li, W. Wang, Y. Dai, N. Niu, J. Lin, Adv. Funct. Mater. 2010, 20, 1166.

[4] A. Gnach, T. Lipinski, A. Bednarkiewicz, J. Rybka, J. A. Capobianco, Chem. Soc. Rev. 2015, 44, 1561.

[5] Z. Cheng, D. L. J. Thorek, A. Tsourkas, Angew. Chem. Int. Ed. 2010, 49, 346.

[6] P. M. Peiris, R. Toy, A. Abramowski, P. Vicente, S. Tucci, L. Bauer, A. Mayer, M. Tam, E. Doolittle, J. Pansky, E. Tran, D. Lin, W. P. Schiemann, K. B. Ghaghada, M. A. Griswold, E. Karathanasis, J. Controlled Release 2014, 173, 51.

[7] G. Y. Lee, W. P. Qian, L. Wang, Y. A. Wang, C. A. Staley, M. Satpathy, S. Nie, H. Mao, L. Yang, ACS Nano 2013, 7, 2078.

[8] H. Wang, Y. B. Sun, J. H. Yi, J. P. Fu, J. Di, A. D. Alonso, S. Q. Zhou, Biomaterials 2015, 53, 117.

[9] X. R. Deng, Y. L. Dai, J. H. Liu, Y. Zhou, P. A. Ma, Z. Y. Cheng, Y. Y. Chen, K. R. Deng, X. J. Li, Z. Y. Hou, C. X. Li, J. Lin, Biomaterials 2015, 50, 154

[10] G. Zhang, J. Gao, J. Qian, D. Cai, K. Zheng, Z. Yu, J. Wang, K. Zhong, X. Zhang, Z. Wu, Part. Part. Syst. Charact. 2014, 31, 976.

[11] Z. Cheng, D. L. J. Thorek, A. Tsourkas, Adv. Funct. Mater. 2009, 19, 3753.

[12] Y. S. Lin, Y. Hung, J. K. Su, R. Lee, C. Chang, M. L. Lin, C. Y. Mou, J. Phys. Chem. B 2004, 108, 15608.

[13] J. Li, X. Shi, M. Shen, Part. Part. Syst. Charact. 2014, 31, 1223.

[14] J. Gao, G. Liang, J. S. Cheung, Y. Pan, Y. Kuang, F. Zhao, B. Zhang, X. Zhang, E. X. Wu, B. Xu, J. Am. Chem. Soc. 2008, 130, 11828

[15] H. Lee, E. Lee, D. K. Kim, N. K. Jang, Y. Y. Jeong, S. Jon, J. Am. Chem. Soc. 2006, 128, 7383.

[16] H. Ai, C. Flask, B. Weinberg, X. Shuai, M. D. Pagel, D. Farrell, J. Duerk, J. Gao, Adv. Mater. 2005, 17, 1949.

[17] S. Xuan, F. Wang, J. M. Y. Lai, K. W. Y. Sham, Y. X. J. Wang, S. F. Lee, J. C. Yu, C. H. K. Cheng, K. C. F. Leung, ACS Appl. Mater. Interfaces 2011, 3, 237.

[18] H. B. Na, T. Hyeon, J. Mater. Chem. 2009, 19, 6267.

[19] H. B. Na, J. H. Lee, K. An, Y. I. Park, M. Park, I. S. Lee, D. H. Nam, S. T. Kim, S. H. Kim, S. W. Kim, K. H. Lim, K. S. Kim, S. O. Kim, T. Hyeon, Angew. Chem. Int. Ed. 2007, 46, 5397.

[20] J. Chen, W. J. Zhang, Z. Guo, H. B. Wang, D. D. Wang, J. J. Zhou, Q. W. Chen, ACS Appl. Mater. Interfaces 2015, 7, 5373.

[21] Y. Li, J. Tang, L. He, Y. Liu, Y. Liu, C. Chen, Z. Tang, Adv. Mater. 2015, 27, 4075 .

[22] C. R. D. Silva, S. Smith, I. Shim, J. Pyun, T. Gutu, J. Jiao, Z. Zheng, J. Am. Chem. Soc. 2009, 131, 6336

[23] R. V. Upadhyay, A. Gupta, C. Sudakar, K. V. Rao, J. Appl. Phys. 2006 99, $08 \mathrm{M} 906$.
[24] S. Sun, H. Zeng, D. B. Robinson, S. Raoux, P. M. Rice, S. X. Wang, G. Li, J. Am. Chem. Soc. 2004, 126, 273.

[25] P. Drake, H. J. Cho, P. S. Shih, C. H. Kao, K. F. Lee, C. H. Kuo, X. Z. Lin, Y. J. Lin, J. Mater. Chem. 2007, 17, 4914.

[26] X. Liang, X. Wang, J. Zhuang, Y. T. Chen, D. S. Wang, Y. D. Li, Adv. Funct. Mater. 2006, 16, 1805

[27] D. Ni, J. Zhang, W. Bu, H. Xing, F. Han, Q. Xiao, Z. Yao, F. Chen, Q. He, J. Liu, S. Zhang, W. Fan, L. Zhou, W. Peng, J. Shi, ACS Nano 2014, 8, 1231

[28] F. Zhang, G. B. Braun, A. Pallaoro, Y. Zhang, Y. Shi, D. Cui, M. Moskovits, D. Zhao, G. D. Stucky, Nano Lett. 2012, 12, 61.

[29] K. Li, D. Ding, D. Huo, K. Y. Pu, N. N. P. Thao, Y. Hu, Z. Li, B. Liu, Adv. Funct. Mater. 2012, 22, 3107.

[30] Y. Xia, T. D. Nguyen, M. Yang, B. Lee, A. Santos, P. Podsiadlo, Z. Tang, S. C. Glotzer, N. A. Kotov, Nat. Nanotech. 2011, 6, 580

[31] Y. Xia, Z. Tang, Chem. Commun. 2012, 48, 6320.

[32] Y. Liu, Z. Tang, Adv. Mater. 2013, 25, 5819.

[33] H. Chu, S. Zhao, K. Yang, J. Zhao, D. Li, G. Li, T. Li, W. Qiao, X. Xu, L. Zheng, J. Xu, Opt. Mater. 2015, 45, 181.

[34] M. Amir, M. Sertkol, A. Baykal, H. SÖzeri, J. Supercond. Nov. Magn. 2015, 28, 2447

[35] Z. Zhou, C. Wu, H. Liu, X. Zhu, Z. Zhao, L. Wang, Y. Xu, H. Ai, J. Gao, ACS Nano 2015, 9, 3012.

[36] T. Paik, T. R. Gordon, A. M. Prantner, H. Yun, C. B. Murray, ACS Nano 2013, 7, 2850.

[37] X. Kang, D. Yang, Y. Dai, M. Shang, Z. Cheng, X. Zhang, H. Lian, P. Ma, J. Lin, Biomater. Sci. 2013, 1, 965.

[38] K. Engin, D. B. Leeper, J. R. Cater, A. J. Thistlethwaite, L. Tupchong, J. D. McFarlane, Int. J. Hyperthermia 1995, 11, 211

[39] Y. Li, Y. Zhou, H. Y. Wang, S. Perrett, Y. Zhao, Z. Tang, G. Nie, Angew. Chem. Int. Ed. 2011, 50, 5860.

[40] X. Li, X. Yang, Z. Lin, D. Wang, D. Mei, B. He, X. Wang, X. Wang, Q. Zhang, W. Gao, Eur. J. Pharm. Sci. 2015, 76, 95.

[41] A. Datta, N. Bhasin, H. Kim, M. Ranjan, B. Rider, Z. Y. A. Elmageed, D. Mondala, K. C. Agrawala, A. B. Abdel-Mageed, Cancer Lett. 2015 $362,25$.

[42] K. S. Kim, W. Park, J. Hu, Y. H. Bae, K. Na, Biomaterials 2014, 35, 337

[43] T. S. Hauck, T. L. Jennings, T. Yatsenko, J. C. Kumaradas, W. C. W. Chan, Adv. Mater. 2008, 20, 3832.

[44] X. Li, X. Yang, Z. Lin, D. Wang, D. Mei, B. He, X. Wang, X. Wang, Q. Zhang, W. Gao, Eur. J. Pharm. Sci. 2015, 76, 95.

[45] G. Zhang, J. Gao, J. Qian, L. Zhang, K. Zheng, K. Zhong, D. Cai, X. Zhang, Z. Wu, ACS Appl. Mater. Interfaces 2015, 7, 14192.

[46] M. Arjomandnejad, A. Muhammadnejad, M. Haddadi, N. SherkatKhameneh, S. Rismanchi, S. Amanpour, S. Muhammadnejad, Arch. Iran. Med. 2014, 17, 273

[47] F. Takahashi-Yanaga, T. Yoshihara, K. Jingushi, Biochem. Pharmacol. $2014,89,340$ 